\title{
On the Integration of Digital Technologies into Mathematics Classrooms
}

\author{
Celia Hoyles, Richard Noss and Phillip Kent \\ Institute of Education, University of London
}

\begin{abstract}
Trouche's (2003) presentation at the Third Computer Algebra in Mathematics Education Symposium focused on the notions of instrumental genesis and of orchestration: the former concerning the mutual transformation of learner and artefact in the course of constructing knowledge with technology; the latter concerning the problem of integrating technology into classroom practice. At the Symposium, there was considerable discussion of the idea of situated abstraction, which the current authors have been developing over the last decade. In this paper, we summarise the theory of instrumental genesis and attempt to link it with situated abstraction. We then seek to broaden Trouche's discussion of orchestration to elaborate the role of artefacts in the process, and describe how the notion of situated abstraction could be used to make sense of the evolving mathematical knowledge of a community as well as an individual. We conclude by elaborating the ways in which technological artefacts can provide shared means of mathematical expression, and discuss the need to recognise the diversity of student's emergent meanings for mathematics, and the legitimacy of mathematical expression that may be initially divergent from institutionalised mathematics.
\end{abstract}

\section{Introduction}

This paper originated as a response (Hoyles, 2003) to the presentation by Luc Trouche (2003) at the Third Computer Algebra in Mathematics Education (CAME) Symposium ${ }^{1}$. Trouche's paper represents another instalment in the formidable sequence of contributions made by French mathematics educators to the developing theory of computationally-mediated mathematical knowledge: see, for example Lagrange (1999), Guin \& Trouche (1999), and also the presentation in 2001 at the Second CAME Symposium by Artigue and the responses by Ruthven and Cuoco (Artigue, 2002; Ruthven, 2002; Cuoco, 2002). The idea of this paper is to

See the CAME website: www.lonklab.ac.uk/came 
provide an interpretation of Trouche's work from our own "Anglo-Saxon" perspective, and to try to connect it - as part of the corpus of French didactical research - with other frameworks and paradigms, and experience with other technologies.

A central focus of this paper, like that of Trouche's, will be on the notions of "instrumental genesis" and "orchestration", the former concerning the mutual transformation of learner and artefact in the course of constructing knowledge with technology; the latter concerning the problem of integrating technology into classroom practice, a problem that still requires considerably more theoretical elaboration and empirical analysis. To this end, we will briefly summarise what we interpret as the main points in the theory of instrumental genesis, and attempt to compare and contrast it with description of the notion of situated abstraction, which the current authors have been developing over the last decade or so. We then seek to broaden the discussion of orchestration so as to elaborate the role of artefacts in the process, and return to the notion of situated abstraction to describe how it might be used in analyses of the evolving mathematical knowledge of both individuals and communities. We conclude by stressing the need to recognise the diversity of students' emergent meanings for mathematics, and to establish the legitimacy of mathematical expression that may (initially at least) be divergent from institutionalised mathematics.

\section{Setting the scene: The marginalisation of technology}

Following a major national effort in France starting in the 1990s to implement the use of mathematical technology (particularly CAS and dynamic geometry) in middle and high school classrooms, there has been a considerable attempt among mathematics education researchers in recent years in France to analyse both theoretically and empirically attempts at integration of technology into classroom practices (see, for example, Artigue, 2000, 2002; Lagrange et al, 2001). Yet despite this level of effort, Artigue (2002) has pointed out that:

...difficulties are indeed persistent in France in spite of the continuous governmental support given to integration for more than 20 years now. ... the complexity of instrumental genesis has been widely under-estimated in research and innovation on [ICT in education], until quite recently. (Artigue, 2002, p. 253)

Artigue (2000) characterises the integration of digital technologies into mathematical education as "marginal" and proposes four main reasons: 


\section{The poor educational legitimacy of computer technologies as opposed to their high social} and scientific legitimacy. Artigue illustrates how the computer's high status and visibility is expected radically to improve learning and teaching, while the values and norms of mathematics teaching and what is to be learned remain essentially invariant. This leads to an inevitable vicious circle of disillusion.

2. The underestimation of issues linked to the computerisation of mathematical knowledge. Complex processes govern the transformation ("transposition" as it is called in the French research) of mathematical knowledge in the classroom context. The computer adds new layers of complexity, which if unrecognised (as has largely been the case to date) can lead to the rejection of mathematical meanings and discourse fostered by computer use, due to the (inevitable) discrepancy between this and the official mathematical discourse of the classroom.

3. The dominant opposition between the technical and conceptual dimensions of mathematical activity, which allows a too-easy characterisation of the computer's role as "automatically" diminishing the former and thus almost "inevitably" enhancing the latter. Trouche, Artigue and other French researchers have carefully documented how attempts at integration which make a simple demarcation of classroom activities into "technical" and "conceptual" — as expressed, for example, in claims that technology will free the student from technical "details" (techniques) in order to focus on mathematical concepts — drastically misjudge the complexity of instrumental genesis.

4. The underestimation of the complexity of instrumentation processes. This is the key issue that we shall address in more detail in what follows. For the time being, this problem can be formulated as a failure adequately to recognise the full extent of the "new" mathematical and technological demands placed on students, and the need to theorise ways to connect to the standard mathematics curriculum, a changed (but, we argue, still mathematical) student body of knowledge.

This set of problems points, in part, to a failure to theorise adequately the complexity of supporting learners to develop a fluent and effective relationship with technology in the classroom - by "effective" we mean helping learners engage with, develop and articulate understandings of mathematical procedures, structures and relationships through the technology and according time and status to this process. Moreover, the values attached to mathematical knowledge can hardly remain invariant under technological transformation; else, as Artigue (2000) puts it, "can the price which needs to be paid in order to transform complex objects into efficient mathematical instruments be justified?" 
The ways in which mathematical knowledge is transformed by the computer's presence have been a preoccupation of ours for many years, beginning with projects with Logo in the 1980s, and we have increasingly come to focus on the epistemological transformations of students' knowledge in interactions with computers in mathematics classrooms (see also Hoyles and Noss, 2003, for a discussion of the types of software that have the potential to afford such transformation). For example, learners' experiences with mathematical variables can be transformed by first encountering them as "inputs" to Logo programs whose values can be easily changed with immediate observable effects; intensive quantities such as rates notoriously difficult because of their abstractness - can be made "objects" to be manipulated in many computer environments; and conjectures can be tested by making constructions in dynamic geometry systems, thus transforming early encounters with proof from procedural exercises in validation to exploratory exercises in explanation. These transformations of mathematical meanings generated in "contexts of integration", necessitate a conception of mathematical understanding and of mathematical knowledge, which properly accounts for the specificity of situations and the contingencies of mathematical expression on tools and technologies and on the communities in which they are used.

This developing view of mathematical knowledge has helped us make sense of activities we have researched in a range of quite different situations: activities with adults in workplaces, as well as with students and digital technologies (Noss and Hoyles, 1992, Hoyles, Noss \& Pozzi, 2001; Noss, Hoyles \& Pozzi, 2002; Kent \& Noss, 2002). We have sought, therefore, to elaborate a unified theoretical account of how conceptions of mathematics might be situated in terms of language and connectivity and with the context of their genesis, means of expression and use (that is, with the artefacts, goals and discourse that form part of the activity) - and yet are abstract in that they extend beyond immediate concerns to more general conceptions of knowledge, that is, they can be "mapped onto" parts of formal mathematics. It is from this basis that we developed the twin theoretical notions of situated abstraction and webbing (see Noss \& Hoyles, 1996), ideas to which we will return below.

The marginalisation of technology by educational institutions has also turned our attention to the need for a more precise analysis of the role of the teacher in these new and changing didactical contexts. As Artigue points out, the computer's legitimacy has often been justified in terms of its ease of use, the relative simplicity of its adoption, and the overstatement of its potential (as if, incidentally, "it" - that is, the computer - is a thing which can be considered unproblematically as an "element" in an otherwise static and unresponsive educational context, 
or which straightforwardly 'affords' this or that entailment). All too often, students are left to their own devices, exploring mathematical tasks for themselves via their computer interactions as teachers struggle to interpret what they do in mathematical terms. Furthermore, the transformations of mathematical knowledge in the presence of technology may or may not be judged as desirable from an educational point of view: it depends on the value of the transformed knowledge as perceived, perhaps differently, by teachers and students. Thus, even though students may be engaged and feel that the activity is legitimate from their point of view, it may still pose a problem of legitimacy for the teacher, since what is expressed by students and how - has to be recognised as mathematical within the discourse of the institutional learning system for mathematics.

\section{Instrumental genesis and situated abstraction}

The analytical framework of the French research is based on the notions of artefact and scheme, where the latter is the psychological component of an instrument, a psychological construct that a person operationalises in activity with an artefact in order to carry out some task. Instrumented activity - i.e. activity that employs and is shaped by the use of instruments has a twofold outcome (Artigue, 2002). There is a process of instrumentalisation, in which (in our terms) the subject shapes the artefact for specific uses, and simultaneously a process of instrumentation, in which the subject is shaped by actions with the artefact. This dialectic by which learner and artefact are mutually constituted in action is referred to as instrumental genesis (see Vérillon, 2000, for a summary of these ideas; also Vérillon \& Rabardel, 1995).

A key challenge, then, for the integration of technology into classrooms and curricula is to understand and to devise ways to foster the process of instrumental genesis. On a theoretical level, Trouche makes good use of the notion of scheme for this task, recognising the possibilities of reconciling the individual and social perspectives most obviously linked to the work of Piaget and Vygotsky, and building on the work of Rabardel and Samurçay (2001). He draws our attention to a still relatively undeveloped area, in which he (and we) are concerned to map the important specificities of mathematical knowledge that are not necessarily part of the general psychological constructs which support "knowledge generation" by individuals.

Although schemes of instrumented action recognise the crucial shaping of the learner by interaction with tools, their very generality makes it all the more important to take account of the specific way mathematical knowledge might be developed. This is what the notion of situated abstraction seeks to address, by providing a means to describe and validate an activity 
from a mathematical vantage point but without necessarily mapping it onto standard mathematical discourse. The notion is particularly pertinent in computational environments, since the process of instrumental genesis involving the new representational infrastructure supported by the computer will tend to produce individual understandings and ways of working that are divergent from standard mathematics.

In fact, both we and the French researchers are acutely sensitive to the discontinuities between computationally mediated mathematical abstraction and the norms of traditional mathematical curricula. Abstraction is a key concern within the field of mathematics education - a characteristic it does not necessarily share with other disciplines - that has been discussed in a variety of contexts by many researchers (such as Arcavi \& Hadas, 2000; Dreyfus, 1993; Nemirovsky et al, 1998). Our contention is that it is the process of abstraction of mathematical properties and invariants that is key, and this is necessarily both situated and shaped by the tools being used, the users' relationship to the tools — including whether the users judge them to be expressive of their developing mathematical meanings — and ultimately whether these meanings are valued and judged by the community (e.g. the classroom) to be mathematical. One of the most remarkable characteristics of digital technologies, such as CAS, is that they provide an unprecedented symbolic means of expression for mathematical abstraction as a process - something which has been wholly embraced by professional users of mathematics (e.g. in science and engineering, the use of multiple representations in the CAS to model and design physical processes, or in mathematics using the visualisation power of a CAS to explore abstract mathematical structures), but which, as we noted at the beginning, remains marginal to the concerns of the mathematics classroom.

It might be helpful at this point to look again at several of the examples presented by Trouche. He presents an example from Guin and Trouche (2002), which demonstrates how the tools provided by a CAS/graphical calculator shape the ways in which a user might solve two equations in two unknowns, and how one may interpret the meanings that the user constructs concerning equations and their solutions. Later, in a discussion of students attempting to find the limit of a function not directly computed by a CAS calculator, he describes how they build specific schemes and notes the differentiation among students' behaviours (our emphasis). These behaviours are then distinguished as different instrumentation processes, which are closely connected with what Trouche calls "command process". We infer from Trouche that the students will develop different instruments. Given that the mathematical goal of the activity is to learn about limits, we are sure that Trouche would agree that students will develop 
different conceptions of limit, and that - in our terms - they are, in general, likely to develop different situated abstractions of the notion of mathematical limit that are intricately connected to their use of the tools.

In moving from an individual to a more social perspective, Trouche distinguishes different facets of the notion of a scheme and mentions its extension beyond the individual to what are termed social schemes. He notes that the notion of social schemes may have certain closeness to situated abstraction, although as far as we can tell, the idea of social schemes, like the schemes of individual cognition, does not in itself take any explicit epistemic focus.

Clearly, it is vital to understand the connections between instrumental genesis (at the student level) and orchestration (of a group of students learning in the classroom). The challenge, therefore, is to theorise the mechanisms by which situated abstractions are developed in and through a community, harnessing the discourse (shaped by the tools at hand) as a means for student-student and student-teacher communication, as well as a means for generating individual knowledge. Thus it is important to keep in mind that the word abstraction refers to both an action (a collective action, in the classroom), and to the intellectual structure (within individuals) that results from that action. More pragmatically, if we could understand more precisely the roles that teachers are called upon to play in computationally-based environments , then it would surely throw light on the nature of the mathematical knowledge involved, its relationship with the priorities of the official mathematics curriculum, and help to clarify how the process of integrating technologies productively into classroom practice might be facilitated.

\section{Orchestration and the role of artefacts}

In Trouche's paper, the term orchestration refers to a process of “external steering of students' instrumental genesis", and through this to enhance their learning of mathematics. Hence orchestration is an element of the broader didactic process of mathematics teaching. Orchestration/instrumentation is a felicitous metaphorical pairing, which captures something of the relationships involved ${ }^{2}$. One striking issue about the metaphor (although bearing in mind that metaphors should not be interpreted too literally) is this: what is the analogue of music in the orchestration of mathematical instruments? Presumably the answer is mathematics, but

\footnotetext{
2 Cf. in music: "Orchestration is the study and practice of arranging music for an orchestra or musical ensemble. In practical terms it consists of deciding which instruments should play which notes in a piece of music." [Source: www.wikipedia.com]
} 
what kind of mathematics? The production of "official" mathematics which the teacher (i.e. conductor) wishes "to hear" in order to judge the students' performance, or the more personal and community-based norms tied to students' own developing conceptions and personal inclinations? This question is not intended to raise general issues of learning style or "epistemological preference" (cf. essay on epistemological pluralism, by Turkle and Papert, 1990), but rather, the complex set of issues which emerge wherever technology has a presence in a mathematics classroom, and the dialogue that must take place between standard ("official") mathematical knowledge, knowledge about the tool, and the "computational transposition" of the mathematical knowledge involved (cf. Balacheff, 1993). For example, Defouad's (2000) research, described in Artigue (2002), illustrates the difficulty with which (CAS-based) instrumented techniques gained mathematical status within a classroom and the manner in which this tended to reduce the value of these techniques. Even when fully legitimated, the techniques retained a sort of intermediate status in the classroom culture: Defouad introduced the notion of "locally official techniques" in order to give an account of this phenomenon. There is no simple way to solve the problem of integrating technology: situated abstractions by their nature are diverse and interlinked with the tools in use, so how can meanings be shared in the classroom, interconnect with each other and with standard mathematical discourse?

The roots of the notion of instrumentation are cognitive, and if instrumented activity is (strictly) judged from this perspective, then the process of instrumentation is one of cognitive "overhead", that is, a process of achieving a mastery over tools that is required in order for a learner to begin to perform the "useful" task of learning mathematics. However, from a sociocultural point of view, instrumentation could be regarded as part of the process of developing participation within a community of practice, a process by which individual understanding and behaviour develops from and contributes to the collective activity.

Trouche describes an example of orchestration in which the architecture and organisation of the classroom is configured to help "connect up" students, with attention to where key students should sit, and which technologies should be switched on or off in order that individual instrumented actions can become the object of collective as well as individual reflection and discussion. This pedagogical strategy has considerable potential and is certainly facilitated by appropriate technical infrastructure ${ }^{3}$. Left relatively unexplored, however, is analysis of the trajectories of students' evolving (instrumented) mathematical knowledge, in terms of the In England, this sort of scenario is nowadays most likely to be around an interactive whiteboard. 
situated abstractions of mathematical ideas that are being developed and expressed, and how these abstractions are webbed by the available tools and shaped by the interactions with these tools and with the community. Further, we need to consider what may be gained by recognising explicitly the relationship between the diversity of individual and community knowledge and the official mathematical knowledge of the curriculum.

Trouche would, we are sure, agree that thus far, the discussion of orchestration and what he terms instrumented orchestration is still schematic. Perhaps the different unfolding histories of our respective research efforts - including the choice of technologies to study - has resulted in differences in emphasis between us rather than in core perspective, most notably regarding the weight we accord to the role of interactions among learners, and the role of technology in mediating these interactions. To assist further in sharpening the discussion, we suggest that it might be essential to bring into any elaboration of the orchestration process an analysis of the ways in which artefacts shape this process, and thus also effect the learning of individuals and communities.

Relevant to this endeavour would be a consideration of the considerable amount of research that has been undertaken on the role of group work in learning mathematics and the importance of forming collaborative communities in classrooms that encourage sharing of different perspectives, explaining and defending ideas and critiquing and debugging the developing ideas of the group (see for example Healy, Pozzi \& Hoyles, 1995). Computer-based collaboration, in particular, has been found to encourage a shift in relationships between teacher and student and - under suitably managed circumstances with appropriate tools enhanced task-based interactions between students and between students and teachers. More generally, there has also been a substantial research effort that seeks to analyse the role of connectivity in teaching and learning (see, for example, the literature on computer-supported collaborative learning, CSCL, in Koschmann, Hall \& Miyake, 2001). A major area of CSCL research concerns knowledge building through shared construction and interaction - to avoid the trap of the didactical paradox, in which students are assumed already to know what it is they are supposed to learn.

A focus on collaborative learning and co-construction of knowledge leads us to envisage a mathematical tool, such as CAS, as not only a cognitive tool but also a genuine mediator of social interaction through which shared expressions can be constructed. Indeed, this goal is now readily technically achievable given that handheld calculators and laptop or palmtop computers can now effortlessly communicate with each other and with other digital devices. It 
would, of course, be wrong to think of any artefact as "just" an artefact - the whole point of instrumental genesis (as well as current socio-cultural theories of learning) leads us to think otherwise. But while it would be wrong to suggest that the meanings of artefacts for students can be drawn directly from the intentions of their designers, it is helpful to think of the possibilities that accrue in the uses by students of artefacts, especially when these artefacts are designed with the intention of promoting learning outcomes for individuals or for groups. The designs of computational tools - and these include the design of individual programs by classroom teachers as well as the "global" design of hardware and software by professional engineers/programmers - constrain the kinds of mathematical expression that is natural to undertake with them, how meanings are constituted and how mathematical ideas are communicated.

We do not mean to imply that the only, or even the primary, mechanism of orchestration is invested in the technology. We do, however, insist that the medium - while not being the message - certainly shapes what messages are natural, and how they might be expressed. We have in the past expressed this by saying that there can be a mathematical epistemology "built into" artefacts, provided we are clear that it is students who breathe life into the technologies and rebuild the mathematical structures for themselves by means of their actions with them. In fact, this is part of the webbing idea, the process by which the student infers meaning by coordinating the structure of the learning system (including the knowledge to be learned, the learning resources available, prior student knowledge and experience and constructing their own scaffolds by interaction and feedback (Noss \& Hoyles, 1996). The idea is that it is the representational infrastructure, that is the artefacts together with the rules of discourse that surround them, that provides a system that learners can draw upon and reconstruct to make mathematical sense.

Thus in our view, the design of tools, activities and tasks is central. Of relevance here is that it is the design of the tools with which individuals interact (i.e. what is and is not easily expressible), and the design by which these tools are connected (i.e. what is and is not easily communicable), which plays an important role in shaping the construction of the sociomathematical norms of the classroom (see for example, Cobb, Stephan, McClain \& Gravemeijer, 2001). Clearly, the teacher is central to this process. We simply wish to raise the profile of the potential role of the technological tools and the kinds of symbolic language and interactions that they might foster among learners. Consider, just as a simple example, a learning environment that typically develops where rows of computers are physically fixed one 
behind the other with little if any connectivity, and contrast this with one where students have wireless-connected laptops and can enjoy a hugely greater freedom to collaborate and share their ideas, both face-to-face and virtually. This process of breathing life into technology necessarily differs from one individual to another, if only because of differences between the kinds of things a student takes for granted, already knows, or is trying to understand. In so far as the artefact's properties can be thought of as "orchestrating" the actions and expressions of an individual or a group of learners, this orchestration is not invariant across different individuals. Viewed in this light, individual difference is clearly not something to be minimised or avoided, it is an inevitable part of orchestration itself.

\section{Situated abstraction revisited}

Recognising the diverse ways in which individuals use and communicate with technologies to express mathematical ideas is not without difficulty. In what follows, we will suggest further that the notion of situated abstraction as a category of mathematical expression may adequately complement the idea of instrumental genesis, while effectively describing the kinds of mathematical knowledge that arise in collective as well as individual instrumented activity.

One helpful starting point is the notion of boundary object (Star, 1989; Star \& Griesemer, 1989). A boundary object names an important class of knowledge artefact, an object which is shared between different communities of practice, which can be used differently by the communities, but can also provide a means to think and talk about an idea without the necessity of any one community adopting the perspective of the other, or even requiring one community to understand the detail of what is already understood by another. This is the key point for meaning-creation: a boundary object provides a generalised mechanism for meanings to be shared and constructed between communities - for example, the community of teachers and the community of students.

From a specifically mathematical point of view, boundary objects can provide the means by which a common discourse involving situated abstractions might be fostered within a community of mathematical learners, as well as a means by which different communities might come to an agreement (often implicitly) that they are talking about "the same" mathematical abstraction or set of abstractions. It does not matter whether or not the abstractions really are the same (indeed, it is a moot question as to what "the same" might mean in this sense). What does matter is that different situated abstractions can be brought into association and alignment through negotiation around a boundary object: between, for example, a community of learners 
engaging in "context-bound" mathematics and the community of teachers of "institutionalised" mathematics. (See Newman, Griffin \& Cole, 1989, for a somewhat similar perspective developed within a Vygotskian, rather than an activity theory framework.)

In the CAS-equipped mathematics classroom, a boundary object might be the display of an artefact (the CAS software) together with the "gestures" required to carry out certain mathematical procedures (Trouche gives some examples in the case of graphics calculators expressing equations to be solved, as discussed earlier). These, we suggest, can be thought of as generative of situated abstractions: students construct and interpret procedures and teachers (re)interpret their constructions. Situated abstraction thus becomes the concrete (visible and audible) expression of the different communities' views of the boundary object.

Viewed in this light, orchestration becomes a mutual act, rather than something that one community does to another. In other words, thinking in terms of boundary objects suggests a kind of orchestration in which mutual negotiation and meaning-construction is the norm for both sides of the boundary, rather than the preserve of one protagonist. In a pedagogic setting, boundary objects are generally carefully designed, not simply objects about which communities happen to coalesce. In the past, many of us who have researched in this area have worked with learners interacting in computer-based microworlds that were designed to foster mathematical meanings through mutual construction, interaction and feedback (see for example Healy, Hoyles and Pozzi, 1995). The idea was that the students could web their own thinking by communicating with and through the tools of the microworld and shaping them to fit their own purposes, including the need to communicate with others. Thus it was through careful design of tools and of the interactions planned to take place in activities around these tools, that we as observers were able to trace and analyse the learners' thinking-in-change their construction of mathematical meanings.

We therefore would like to suggest that the notion of situated abstraction might complement the instrumentation-orchestration theory, by focusing specifically on the abstraction process. Orchestrating to foster situated abstractions as well as to negotiate common meanings is a key objective of teaching. An important challenge therefore lies in finding the right kinds of representational infrastructure (tools and linguistic frameworks) for computational environments, which highlight (to the student) the key mathematical elements of a problem situation and provide "natural" expressive power - the right things to talk about, and ways to talk about them. 


\section{Conclusions}

Research in mathematics education evolves through the design of classroom interventions, feeding back into theoretical analysis. The notion of engineering as described in French didactics is powerful: it suggests that the artefact (in this case, the didactical ${ }^{4}$ artefact) exists in terms of a specified outcome. This has proved helpful since a general goal of mathematics education research is to be able to specify the engineering of situations which, taken together on a reasonable time scale, will enhance mathematical learning. A similar notion to didactical engineering that we find appealing is that of "design experiments" — see for example see Cobb, Confrey, diSessa, Lehrer, \& Schauble, 2003. However, on the shorter temporal scale that typically governs change within the learning domain of an individual classroom teacher, we suggest that Trouche's perspective might benefit from a somewhat more explicit acknowledgement of the diversity of student (and teacher) response, and by implication, the need to orchestrate at much higher levels of the system. .

Our two perspectives are united in recognising that if orchestration aims merely to bring about "convergence" of mathematical expression with official mathematical discourse, the potential of the technology will almost certainly be missed. Yet convergence is important, so two qualifications of the previous comment are in order. First, convergence for the students may take time - significant time over years rather than days or months. Second, the process of orchestration must take place at different levels, separated in time: the first level of orchestration being to foster the growth of situated abstractions (or, instrumented social and individual mathematical schemes) which establish a "cognitive scaffolding" for a second level of orchestration to bring about convergence or at least alignment through discussion of boundary objects. This second phase might be expected to take place over an extended period, and through a combination of collective activity in the classroom and individual work by students. We suggest that a fundamental conjecture of Seymour Papert might usefully be revisited with the insights provided by the theoretical apparatus we have tried to elaborate: namely, that computationally-based activity should be seen, in the first stage, as a preparation for this kind of convergence rather than a means of effecting it: indeed, Papert argued convincingly that a key outcome of such activity is that it should make it "easier" at the later point where formal teaching takes place (Papert, 1972). 
The collective French research effort, exemplified by Trouche's paper, has provided a theoretical way forward, and offers the notion of orchestration as central in the construction of effective teaching and learning environments from the design point of view. As a next step, the research community could usefully identify and present scenarios in use (as mentioned by Trouche) with the inclusion of analyses of the trajectories of students' evolving situated abstractions and how they are shaped and become taken-as-shared as a result of didactical strategies followed by the teacher (see Healy, 2002, on "filling out" and "filling in" as a systematic example of this approach). This might be one way to understand better the instrumentation-orchestration process and to tease out the implications for classroom activity. Our intention in this paper has, therefore, been to expand the notion of orchestration to include a cultural dimension, a recognition that it is not merely the direct teacher-student interaction that needs to be engineered, but also the "medium" in which this interaction takes place, and above all the artefacts and objects that are shared between the communities of teachers and learners.

We conclude with two important caveats. First, we recognise that pedagogy is closely tied to cultural contexts and that the French and Anglo-Saxon mathematical cultures are distinctly different. Perhaps there is a case for collective investigation through design experiments or didactical engineering projects in several countries, which take our combined theories as a starting point. We certainly do not presume to imagine that the challenges that face UK mathematics educators map precisely onto those faced by our French colleagues, even though we $d o$ believe that the four key problems outlined by Artigue at the outset of this paper are indeed those that are generally faced by us all.

Second, we would like to caution against epistemological relativism, which our advocacy of diversity may seem to suggest. Mathematical legitimacy is important to us. It is not denied by recognising that there exists a diversity of knowledge webs in which people make connections as a result of new computational tools: they see things they couldn't see; connect things that couldn't be connected; represent things that were hitherto un-representable. Connection always a key mathematical objective - becomes a possibility. But connectivity is not a strict sequence of steps that lead from disconnection to connection. Recognising the diversity of webs, and their development over relatively long timescales, is key to bringing about

\footnotetext{
$4 \quad$ We borrow the French formulation here. Simply, didactic $=$ pedagogy + knowledge. At a time when, in AngloSaxon cultures at least, the epistemological dimension is so widely taken for granted, this formulation is helpful, even though the connotations of the word "didactic" in English are potentially misleading.
} 
International Journal of Computers for Mathematical Learning 9, 3, 309-326

legitimation of students' evolving knowledge, and a legitimation of the role of digital technologies in the mathematics classroom.

\section{Acknowledgements}

We would like to thank Michèle Artigue and Lulu Healy for their comments on earlier versions of this paper. We are grateful to the participants of the CAME Symposium in Reims for helping to crystallise some of the ideas presented here.

\section{References}

Arcavi, A. \& Hadas, N. (2000). "Computer mediated learning: and example of an approach". International Journal of Computers for Mathematical Learning, 5, 1, 25-45.

Artigue, M. (2000). "Instrumentation Issues and the Integration of Computer Technologies into Secondary Mathematics Teaching". Proceedings of the Annual Meeting of the GDM, Potsdam, 2000. [download: http://webdoc.sub.gwdg.de/ebook/e/gdm/2000]

Artigue, M. (2002). 'Learning Mathematics in a CAS environment: The Genesis of a Reflection about Instrumentation and the Dialectics between Technical and Conceptual work'. International Journal of Computers for Mathematical Learning, 7, 3, 245-274.

Balacheff, N. (1993). “Artificial intelligence and real teaching”. In C. Keitel \& K. Ruthven (Eds.), Learning from computers: Mathematics education and technology pp. 131-158. Berlin: Springer-Verlag.

Cobb, P., Stephan, M., McClain, K., \& Gravemeijer, K. (2001). "Participating in classroom mathematical practices". Journal of the Learning Sciences, 10, 113-164.

Cobb, P., Confrey, J., diSessa, A., Lehrer, R., \& Schauble, L. (2003). “Design Experiments in Educational Research". Educational Researcher, 32, 1, 9-13.

Cuoco, A. (2002) “Thoughts on Reading Artigue's 'Learning Mathematics in a CAS Environment"'. International Journal of Computers for Mathematical Learning, 7, 3, 293 291.

Defouad, B. (2000) Etude de genese instrumentals liees a l'utilisation d'une calculatrice symbolique en classe de premiere These de doctorat, Universite Paris 7. 
International Journal of Computers for Mathematical Learning 9, 3, 309-326

Dreyfus, T. (1993), “Didactic Design of Computer-based Learning Environments”, in C. Keitel \& K. Ruthven (Eds.), Learning from Computers: Mathematics Education and Technology, (NATO ASI Series F, vol 121) pp. 101-130. Berlin: Springer-Verlag.

Guin, D. and Trouche, L. (1999). "The complex process of converting tools into mathematical instruments: The case of calculators". International Journal of Computers for Mathematical Learning, 3, 3, 195-227

Healy, S., (2002) Iterative design and Comparison of Learning Systems for Refection in Two Dimensions. Unpublished PhD thesis, University of London Institute of Education.

Healy, L., Pozzi, S., \& Hoyles, C., (1995). "Making sense of groups, computers and mathematics". Cognition and Instruction, 13 (4), 505-523.

Hoyles, C. (2003). "From Instrumenting and Orchestrating Convergence to Designing and Recognising Diversity: A response to Luc Trouche". Plenary presentation at the Third Computer Algebra in Mathematics Education Symposium, Reims, France, June 2003.

Hoyles, C. and Noss, R. (2003) 'What can digital technologies take from and bring to research in mathematics education?' In A.J. Bishop, M.A. Clements, C. Keitel, J. Kilpatrick and F.K.S. Leung (eds), Second International Handbook of Mathematics Education. 323-349. Dordrecht: Kluwer Academic Publishers.

Hoyles, C., Noss, R. and Pozzi S. (2001). "Proportional Reasoning in Nursing Practice". Journal for Research in Mathematics Education 32, 14-27.

Kent, P. and Noss, R. (2002). The Mathematical Components of Engineering Expertise (End of project report). London: Institute of Education. [www.ioe.ac.uk/rnoss/MCEE]

Koschmann, T., Hall, R. \& Miyake, N. (Eds.) (2001). CSCL2: Carrying Forward the Conversation. Mahwah, NJ: Lawrence Erlbaum

Lagrange, J. B. (1999). “Complex calculators in the classroom: Theoretical and practical reflections on teaching pre-calculus", International Journal of Computers for Mathematical Learning, 4, 1, 51-81

Lagrange, J. B. et al. (2001). “A meta study on IC technologies in education”. in, M. van den Heuvel-Panhuizen. Proceedings of the 25th Conference of the International Group for the Psychology of Mathematics Education, vol. 1 (pp. 111-122). Utrecht: Freudenthal Institute.

Nemirovsky, R., Tierney, C., \& Wright, T. (1998). “Body Motion and Graphing”. Cognition and Instruction, 16 (2), 119 - 172. 
International Journal of Computers for Mathematical Learning 9, 3, 309-326

Newman, D., Griffin, P. and Cole, M. (1989). The Construction Zone: Working for cognitive change in school. New York: Cambridge University Press.

Noss R., \& Hoyles, C. (1996). Windows on Mathematical Meanings: Learning Cultures and Computers, Dordrecht: Kluwer Academic.

Noss, R., Hoyles, C. and Pozzi, S. (2002). “Abstraction in Expertise: A Study of Nurses' Conceptions of Concentration". Journal for Research in Mathematics Education 33(3), 204-229.

Papert, S. (1972). "Teaching Children to Be Mathematicians vs. Teaching About Mathematics". International Journal of Mathematics Education in Science and Technology. Vol. 3, 249-262.

Rabardel, P. \& Samurçay, R. (2001). "From Artefact to Instrumented-Mediated Learning". New Challenges to Research on Learning: An international symposium organized by the Center for Activity Theory and Developmental Work Research, University of Helsinki, March 21-23.

Roschelle (1992). "Learning by collaboration: Convergent conceptual change". The Journal of the Learning Sciences 2 (3), 235-276.

Ruthven, K. (2002) "Instrumenting Mathematical Activity: Reflections on Key Studies of the Educational Use of Computer Algebra Systems". International Journal of Computers for Mathematical Learning, 7(3) pp. 275 - 291.

Star, S. L. (1989). "The structure of ill-structured solutions: Boundary objects and heterogeneous distributed problem-solving”. In L. Gasser \& M. N. Huhns (Eds.), Distributed Artificial Intelligence, volume 2. London: Pitman / San Mateo, CA: Morgan Kaufmann.

Star, S. L. and Griesemer, J. (1989). “Institutional Ecology, 'Translations,' and Boundary Objects: Amateurs and Professionals in Berkeley's Museum of Vertebrate Zoology, 19071939," Social Studies of Science, 19: 387-420.

Trouche, L. (2003). "Managing the Complexity of Human/Machine Interaction in a Computer Based Learning Environment: Guiding Student's Process Command through Instrumental Orchestrations". Plenary presentation at the Third Computer Algebra in Mathematics Education Symposium, Reims, France, June 2003. 
International Journal of Computers for Mathematical Learning 9, 3, 309-326

Turkle, S. and Papert, S. (1991). "Epistemological pluralism and the revaluation of the concrete”. In I. Harel \& S. Papert (Eds.), Constructionism. Norwood, NJ: Ablex Publishing Corp. Pages 161-191. [Online version:

http://www.papert.com/articles/EpistemologicalPluralism.html ]

Vérillon, P. (2000). "Revisiting Piaget and Vygotsky: In Search of a Learning Model for Technology Education”. The Journal of Technology Studies XXVI, 1.

[download: http://scholar.lib.vt.edu/ejournals/JTS/Winter-Spring-2000/]

Vérillon, P. \& Rabardel, P. (1995). 'Cognition and Artefacts: a contribution to the study of thought in relation to instrumented activity', European Journal of Psychology of Education, $10(1)$, pp. 77-101.

Celia Hoyles, Richard Noss AND PhILliP KenT

SCHOOL OF MATHEMATics, SCIENCE AND TECHNOLOGY

INSTITUTE OF EDUCATION

20 BEDFORD WAY

LONDON WC1H OAL

UNITED KINGDOM

c.hoyles@ioe.ac.uk, $\underline{\text { r.noss@ @ioe.ac.uk, p.kent@ioe.ac.uk }}$ 